\title{
DIOPHANTINE CORRECT MODELS OF ARITHMETIC
}

\author{
L. LIPSHITZ
}

\begin{abstract}
A countable nonstandard model of arithmetic is diophantine correct if and only if it can be embedded in arbitrarily short nonstandard initial segments of itself.
\end{abstract}

A model $M$ of Peano arithmetic is called diophantine correct if the same diophantine equations have solutions in $M$ and $\mathbf{N}$ (the natural numbers). The purpose of this note is to prove the following theorem, which was suggested to the author by $\mathrm{S}$. Tennenbaum.

TheOREM. Let $M$ be a countable nonstandard model of Peano arithmetic. Then $M$ is diophantine correct if and only if for every nonstandard $\lambda \in M$ there is an embedding of $M$ into the initial segment $[0, \lambda]=\{x \in M \mid x \leqslant \lambda\}$.

The reader will notice that the existence of such embeddings for aribtrary countable models of Peano arithmetic would have given a positive solution for Hilbert's tenth problem. The proofs of the Theorem and the Remark below are similar to the proof of Theorem 4.2 of [1].

Proof of THE THEOREM. If $M$ is not diophantine correct then there is a polynomial $p\left(x_{1}, \ldots, x_{n}\right)$ with integer coefficients such that $p\left(x_{1}, \ldots, x_{n}\right)$ has a solution in $M$ but has no solution in $\mathbf{N}$. Choose $\lambda \in M$ nonstandard so that $p\left(x_{1}, \ldots, x_{n}\right)=0$ has no solution with $x_{1}, \ldots, x_{n}<\lambda$. Then there is no embedding of $M$ into the initial segment $[0, \lambda]$. Conversely suppose that $M$ is countable and diophantine correct. Let $M=\left\{a_{1}, a_{2}, \ldots\right\}$, and let $\lambda \in M$ be nonstandard. Notice that for any polynomial $p\left(x_{1}, \ldots, x_{n}\right)$ (with integer coefficients) either $p\left(x_{1}, \ldots, x_{n}\right)=0$ has only finitely many solutions, which are all standard, or for every nonstandard $\gamma$ there is a nonstandard solution less than $\gamma$. We shall define partial embeddings $\rho_{n}:\left\{a_{1}, \ldots, a_{n}\right\} \rightarrow[0, \lambda]$ by induction so that for all $n \in \omega$ (i) $\rho_{n} \subset \rho_{n+1}$ and (ii) for every polynomial $p\left(x_{1}, \ldots, x_{n}, y_{1}, \ldots, y_{k}\right)$ if $\exists y_{1}, \ldots, y_{k}\left[p\left(a_{1}, \ldots, a_{n}, y_{1}, \ldots, y_{k}\right)=0\right]$ then $\exists y_{1}, \ldots, y_{k<\lambda}\left[p\left(\rho_{n}\left(a_{1}\right), \ldots, \rho_{n}\left(a_{n}\right), y_{1}, \ldots, y_{k}\right)=0\right]$. We take $\rho_{0}=\varnothing$. Suppose that $\rho_{n}$ is defined and has properties (i) and (ii). Consider the following formula $\psi(m)=\exists z_{<\lambda}$ [for all polynomials $p\left(x_{1}, \ldots, x_{n}, u\right.$, $\left.y_{1}, \ldots, y_{k}\right)$ of length $\leqslant m$ if $\exists y_{1}, \ldots, y_{k}\left(p\left(a_{1}, \ldots, a_{n}, a_{n+1}, y_{1}, \ldots, y_{k}\right)=\right.$ 0) then $\left.\exists y_{1}^{\prime}, \ldots, y_{k<\lambda}^{\prime} p\left(b_{1}, \ldots, b_{n}, z, y_{1}^{\prime}, \ldots, y_{k}^{\prime}\right)=0\right]$, where $b_{i}=\rho_{n}\left(a_{i}\right)$.

Received by the editors May 25, 1977.

AMS (MOS) subject classifications (1970). Primary 02H20, $10 \mathrm{~N} 15$.

${ }^{1}$ Supported in part by National Science Foundation Grant MCS76-06357. 
Using the fact that a system of polynomial equations $\left(\bigwedge_{i} p_{i}(x)=0\right)$ is equivalent to one polynomial equation $\left(\Sigma_{i}\left(p_{i}(x)\right)^{2}=0\right)$ it follows from property (ii) for $\rho_{n}$ that $M \vDash \psi(m)$ for each $m \in \omega$. Hence for some nonstandard $\gamma, M \vDash \psi(\gamma)$. Let $b_{n+1}$ be a corresponding $z<\lambda$, and set $\rho_{n+1}=\rho_{n} \cup\left\{\left\langle a_{n+1}, b_{n+1}\right\rangle\right\}$. It is clear that $\rho=\cup_{n<\omega} \rho_{n}$ is the required embedding.

REMARK. In the above we could have arranged that $\rho$ be an isomorphism of $M$ onto an initial segment of $M$ contained in $[0, \lambda]$. Let $[0, \lambda]=\left\{c_{1}, c_{2}, \ldots\right\}$. Define $\rho$ satisfying (i) and (ii) above by induction as follows. If $\rho_{2 n}$ is defined obtain $\rho_{2 n+1}$ from $\rho_{2 n}$ in the same way that $\rho_{n+1}$ was obtained from $\rho_{n}$ above. Suppose that $\rho_{2 n+1}$ is defined. Let $b$ be the first of the $c_{i}$ 's not in the range of $\rho_{2 n+1}$ but less than something in the range $\left(\rho_{2 n+1}\left(a_{i}^{\prime}\right)=b_{i}\right.$ say). (If no such $c_{i}$ exists let $\rho_{2 n+2}=\rho_{2 n+1}$.) Let $\psi^{\prime}(m)=\exists z_{<a_{i}^{\prime}}$ [for any polynomial $p$ of length $\leqslant m$ if $\neg \exists y_{1}, \ldots, y_{k<\lambda}\left(p\left(b_{1}, \ldots, b_{l}, b, y_{1}, \ldots, y_{k}\right)=0\right)$ then $\neg \exists y_{1}, \ldots$, $\left.y_{k}\left(p\left(a_{1}^{\prime}, \ldots, a_{l}^{\prime}, z, y_{1}, \ldots, y_{k}\right)=0\right)\right]$, where $\rho_{2 n+1}=\left\{\left\langle a_{j}^{\prime}, b_{j}\right\rangle \mid j=\right.$ $1, \ldots, l\}$. Next we shall show that for each $m \in \omega, M \vDash \psi^{\prime}(\underline{m})$. Let $p_{1}, \ldots, p_{r}$ be the polynomials of length $\leqslant_{m}$ such that $M \vDash \neg \exists \bar{y}_{<\lambda} p_{j}(\bar{b}, b, \bar{y})$ $=0$ for $j=1, \ldots, r$ where $\bar{b}=\left(b_{1}, \ldots, b_{l}\right), \bar{y}=\left(y_{1}, \ldots, y_{k}\right)$ and $\bar{a}=$ $\left(a_{1}^{\prime}, \ldots, a_{l}^{\prime}\right)$. Then $M \vDash \exists_{z<b_{i}} \forall \bar{y}_{<\lambda} \bigwedge_{j=1}^{r}\left[\bar{p}_{j}(\bar{b}, z, \bar{y}) \neq 0\right]$. By the results of [2] and the fact that these results can be formalized in Peano arithmetic there is a polynomial $p(\bar{b}, \bar{u}, \bar{v})$ such that $M \vDash \exists z_{<w_{i}} \forall \bar{y}_{<\lambda} \bigwedge_{j=1}^{r}\left[p_{j}(w, z, y) \neq 0\right] \leftrightarrow$ $\neg\left\{\exists \bar{u}, \bar{v}(p(\bar{w}, \bar{u}, \bar{v})=0) \wedge \forall z_{<w_{i}}\left((\bar{u})_{z}<\lambda\right)\right\}$ and $M \vDash \exists z_{<w_{i}} \forall \bar{y} \bigwedge_{j=1}^{r}$ $p_{j}(\bar{w}, z, \bar{y}) \neq 0 \leftrightarrow \neg \exists \bar{u}, \bar{v}(p(\bar{w}, \bar{u}, \bar{v})=0)$ where $\bar{w}=\left(w_{1}, \ldots, w_{l}\right)$. Hence $M \vDash \neg\left\{\exists \bar{u}, \bar{v}(p(\bar{b}, \bar{u}, \bar{v})=0) \wedge \forall z_{<b_{i}}(\bar{u})_{z}<\lambda\right\}$ and hence $M \vDash$ $\neg \exists \bar{u}, \bar{v}_{<\lambda}(p(\bar{b}, \bar{u}, \bar{v})=0)$. By property (ii) this gives us $M \vDash \neg \exists \bar{u}, \bar{v}(p(\bar{a}$, $\bar{u}, \bar{v})=0)$ and thus $M \vDash \exists z_{<a_{i}^{\prime}} \forall \bar{y} \bigwedge_{j=1}^{r}\left[p_{j}(\bar{a}, z, \bar{y}) \neq 0\right]$, i.e. $M \vDash \psi^{\prime}(\underline{m})$. Hence $M \vDash \psi^{\prime}(\gamma)$ for some nonstandard $\gamma \in M$. Let $a^{\prime}$ be a corresponding value of $z$ and set $\rho_{2 n+2}=\rho_{2 n+1} \cup\left\{\left\langle a^{\prime}, b\right\rangle\right\}$. It is clear that $\rho_{2 n+2}$ has properties (i) and (ii) and that $\rho=\cup_{n<\omega} \rho_{n}$ is the required isomorphism.

\section{REFERENCES}

1. Harvey Friedman, Countable models of set theories, Lecture Notes in Math., vol. 337, Springer-Verlag, Berlin and New York, 1973, pp. 539-573.

2. Yuri Matijasevič, Enumerable sets are diophantine, Dokl. Akad. Nauk SSSR 191 (1970), 279-282 = Soviet Math. Dokl. 11 (1970), 354-357.

School of Mathematics, Institute for Advanced Study, Princeton, New Jersey 08540

Department of Mathematics, PURdue University, West Lafayette, Indiana 47907 